\title{
Effects of the airwave in time-domain marine controlled-source electromagnetics
}

\author{
Jürg Hunziker ${ }^{1}$, Evert Slob ${ }^{1}$, and Wim Mulder ${ }^{2}$
}

\begin{abstract}
In marine time-domain controlled-source electromagnetics (CSEM), there are two different acquisition methods: with horizontal sources for fast and simple data acquisition or with vertical sources for minimizing the effects of the airwave. Illustrations of the electric field as a function of space and time for various source antenna orientations, based on analytical formulation of the electric field in two half-spaces, provide insights into the properties of the airwave and the nature of diffuse electric fields. Observing the development of the electric field over time and space reveals that diffusive fields exhibit directionality. Therefore, techniques that have thus far mostly been applied to wavefields can be adapted for CSEM. Examples range from the well-known up-down decomposition to beam steering. Vertical sources have the advantage of not creating an airwave. On the other hand, it is quite difficult to achieve perfect verticality of the source antenna. Results, using a numerically modeled data set to analyze the impact of the airwave on a signal from a subsurface reservoir in the case of a slightly dipping vertical source, indicate that already for a dip of $0.05^{\circ}$, the airwave contributes $20 \%$ to the complete electric field in our configuration of reservoir depth, water thickness, and conductivity values.
\end{abstract}

\section{INTRODUCTION}

Controlled-source electromagnetics (CSEM) in a marine environment can be used to detect resistors, e.g., hydrocarbon reservoirs, in the subsurface. A historical overview of the method is given by Constable (2010). However, the recorded signal can be strongly affected by the airwave, which consists of energy refracted at the air-water interface, traveling through the air at the speed of light and continuously transmitting energy downward into the water. Except for geometric spreading, the airwave signal is not attenuated while traveling in the air (Wait, 1960; Bannister, 1984; King et al., 1992; Amundsen et al., 2006). Thus, the strength of the airwave depends mainly on its vertical diffusion path in the water (Um and Alumbaugh, 2007). Consequently, if the source and receiver antennas are located far from the air-water interface, the airwave is weak.

Because the airwave produces a strong signal that masks the response from a subsurface resistor, one wants to eliminate it from the data during processing. In shallow water, it is possible that energy first interacts with the air-water interface to form an airwave and then interacts with the reservoir. Such an airwave signal contains information about a possible subsurface resistor (Mittet, 2008), but this information content is difficult to extract and interpret. For frequency-domain CSEM, different approaches exist to minimize the effects of the airwave from the data. These approaches include modeling and adjacent subtraction (Nordskag and Amundsen, 2007), weighted differences between pairs of receivers or sources (Løseth et al., 2010; Chen and Alumbaugh, 2011), taking the frequency derivative (Maaø and Nguyen, 2010; Chen and Alumbaugh, 2011) or a difference between fields at two frequencies that are further apart (Wirianto et al., 2011), magnetotelluric impedance stripping (Chen and Alumbaugh, 2011), wavefield decomposition (Amundsen et al., 2006), and interferometry (Wapenaar et al., 2008).

Weiss (2007) shows that the CSEM time-domain response from a subsurface resistor in shallow water may be easier to distinguish from the airwave arrival than in the frequency domain because of the possible separation of the two events in time. In the case of overlapping events, the airwave can be removed by filtering (Ziolkowski and Wright, 2007). Some airwave-removal methods for frequency-domain CSEM may also be applicable for time-domain CSEM.

Manuscript received by the Editor 25 August 2010; revised manuscript received 10 March 2011; published online 7 June 2011.

${ }^{1}$ Delft University of Technology, Applied Geophysics and Petrophysics, Department of Geotechnology, Delft, Netherlands. E-mail: j.w.hunziker@ tudelft.nl; e.c.slob@tudelft.nl.

${ }^{2}$ Delft University of Technology, Applied Geophysics and Petrophysics, Department of Geotechnology, Delft, Netherlands and Shell Global Solutions International, Rijswijk, Netherlands. E-mail: wim.mulder@shell.com.

(C) 2011 Society of Exploration Geophysicists. All rights reserved. 
In this paper, we investigate the effects of the airwave for time-domain CSEM. Two different offshore CSEM methods are available in the time domain. The first uses horizontal sources and receivers (Chave et al., 1991; Ziolkowski, 2007). The processing scheme proposed by Ziolkowski (2007) deconvolves the recorded signal with the source signal, a pseudorandom binary sequence, to recover the earth impulse response. In contrast, the second method, described by Holten et al. (2009), uses vertical sources and receivers. In this method, the source signal consists of eight square pulses followed by silent periods. The signal is stacked over these eight pulses to reduce noise.

The advantage of using vertical sources and receivers is the complete absence of the airwave. Additionally, by using numerical models, Constable and Weiss (2006) show that, in contrast to CSEM with horizontal receivers, vertical receivers are more sensitive to the edges of a subsurface resistor. Consequently, measurements of the vertical electric field are more suitable for mapping the lateral extent of a resistor than recordings of the horizontal electric field.

Unfortunately, acquisition with vertical sources and receivers also has disadvantages. First, a vertical source cannot be dragged through the water behind a boat. This reduces the acquisition speed dramatically. Second, because of currents, seabed topography, and other effects, it can be quite difficult to manage a perfect vertical orientation of sources and receivers. And third, the amplitude of the signal is lower compared to CSEM with horizontal sources and receivers (Holten et al., 2009).
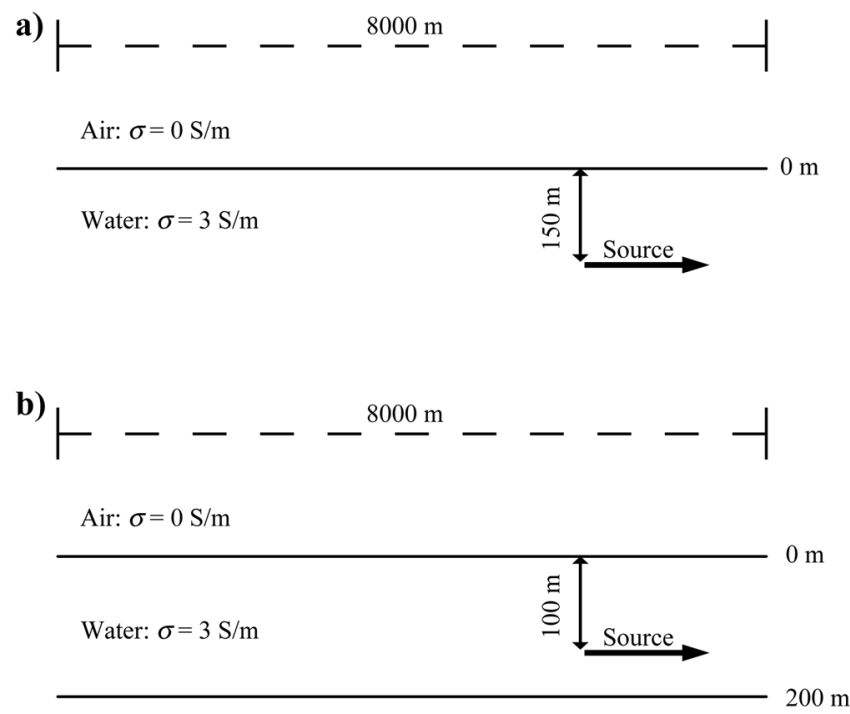

Ground: $\sigma=0.5 \mathrm{~S} / \mathrm{m}$

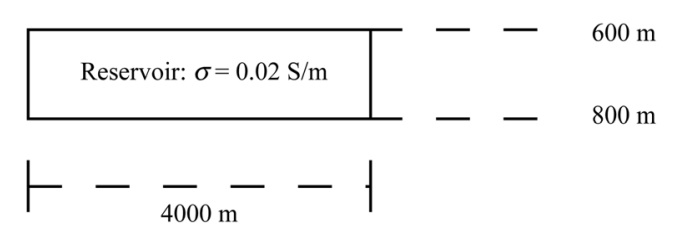

Figure 1. Modeling setups for the (a) analytical and (b) numerical models (not to scale).
The goals of this paper are to illustrate the appearance and the properties of the airwave in marine CSEM and to investigate the effects of imperfect verticality of the antennas when using vertical sources. To analyze the properties of the airwave, we model the electric field analytically in a half-space of water below a half-space of air. Then we use a numerically modeled electric field in a more complicated model with a finite-length reservoir in the subsurface to investigate the effects of imperfect verticality of the source antenna.

\section{METHOD}

We analytically model the electric field in a homogeneous half-space of water (conductivity $\sigma=3 \mathrm{~S} / \mathrm{m}$ ) below a half-space of air $(\sigma=0 \mathrm{~S} / \mathrm{m})$. The source is located $150 \mathrm{~m}$ below the airwater interface. The setup of the analytical model is summarized in Figure 1a.

Raiche and Coggon (1975) give the expressions for the electric field in the lower half-space in the space-frequency domain. Here, we use a space-time-domain expression given by Slob et al. (2010):

$$
\begin{aligned}
E_{k r}= & P_{k r} \\
& +\sum_{m=0}^{2}\left(g_{k r ; m}^{\mathrm{TE} ;-} F_{m}^{-}+g_{k r ; m}^{\mathrm{TE} ;+} F_{m}^{+}+g_{k r ; m}^{\mathrm{TM} ;-} F_{m}^{-}+g_{k r ; m}^{\mathrm{TM} ;+} F_{m}^{+}\right),
\end{aligned}
$$

where $k$ indicates the receiver orientation and $r$ indicates the source orientation $x, y$, and $z$ (or inline, crossline, and vertical directions, respectively). There are five terms on the right-hand side of Equation 1. The first term $P_{k r}$ describes the effects of the airwave in the subsurface and contains modified Bessel functions of integer order. The other four terms consist of a coefficient $g_{k r ; m}$ multiplied with an exponential time-dependent diffusion function $F_{m}^{ \pm}$The first part of the superscripts TE or TM of the coefficient indicates if the corresponding term describes the transverse electric (TE) mode part or the transverse magnetic (TM) mode part of the electric field, respectively. The minus or plus sign in the second part of the superscript indicates the direct and reflected (via the interface) travel paths, respectively.

In other words, the electric field consists of five parts: the airwave effect, the direct and reflected TE-mode parts, and the direct and reflected TM-mode parts. If either the source or the receiver or both are vertical, the coefficients for the TE mode are zero because in that case there is no TE mode. Furthermore, $P_{k r}$ is also zero, which means that no airwave exists for these source/receiver combinations. All coefficients and diffusion functions are derived in Slob et al. (2010) for a VTI medium and are summarized and simplified for an isotropic half-space in Appendix A.

In Appendix B, we give an approximation for the Bessel functions in the airwave term $P_{k r}$ for early times and one for late times. The combination of both approximations in the appropriate time window results in a normalized error no larger than $4 \%$. However, in all of our computations, we use the expression for the airwave, including the Bessel functions and thus without approximations. 
Each of the three source orientations $-x, y$, and $z-$ is modeled for all three receiver components for different depths, horizontal offsets, and time steps. As a result of reciprocity, $E_{x y}$ and $E_{y x}$ are identical. Fields $E_{x z}$ and $E_{z x}$ as well as $E_{y z}$ and $E_{z y}$ differ from each other only in the sign of the reflected field (Raiche and Coggon, 1975; Slob et al., 2010). Although the current dipole source is modeled as a point, i.e., no physical length, different antenna orientations can be simulated by modeling just one component of the source. In the following, a vertical source actually means a vertically oriented point-dipole source. The source-time function is a Dirac delta function.

To investigate the interference of the airwave with a subsurface response, depending on the dip of a vertical source, a slightly more complicated setup is modeled. This setup consist of three layers, in which the last contains a reservoir with finite extent. The geometry and electrical parameters are shown in Figure 1b. The electric field in this setup is modeled numerically using a frequency-domain multigrid code (Mulder, 2006). Modeling is done for several frequencies, followed by an interpolation and a fast Fourier transform (Mulder et al., 2008).

The data cubes are combined geometrically to simulate new source-antenna orientations for both modeling procedures. The orientation of an antenna is defined using two angles: angle $\alpha$ describes dips of the antenna relative to the vertical coordinate axis $z$ and angle $\beta$ describes the azimuth relative to the horizontal coordinate axis $x$ (Figure 2). For horizontal, inline-directed receivers, the data cubes can be combined to form any sourceantenna orientation using the following formulation:

$$
E_{x s}=E_{x x} \cos (\beta) \sin (\alpha)+E_{x y} \sin (\beta) \sin (\alpha)+E_{x z} \cos (\alpha),
$$

where the subscript $s$ in $E_{x s}$ indicates any arbitrary source-antenna orientation. The fields $E_{x x}, E_{x y}$, and $E_{x z}$ are inline-component ( $x$ direction) electric fields from a unit source antenna in the $x$-, $y$-, and $z$-directions, respectively. The same is done for the crossline (y-direction) and the vertical receiver component (z-direction) of the electric field, which are then added up to the total electric field using

$$
E_{t s}=\sqrt{E_{x s}^{2}+E_{y s}^{2}+E_{z s}^{2}},
$$

where the subscript $t$ represents the total electric field on the receiver side. With this procedure, any source orientation can be created and used to analyze the impact of the airwave on the data as a function of the source-antenna orientation.

\section{RESULTS}

\section{Point source}

Figure 3 shows the development of the electric field over time in a half-space of water (see Figure 1a) after a vertical source $\left(\alpha=\beta=0^{\circ}\right)$ at a depth of $150 \mathrm{~m}$ is excited. Five time slices between $10^{-3} \mathrm{~s}$ and $10^{1} \mathrm{~s}$ are displayed. The source is modeled as a point-dipole source in space and time (Dirac delta function). The left column contains vertical cross sections as a function of depth and inline offset, whereas the right column contains horizontal cross sections as a function of inline and crossline offset. The color represents the amplitude of the total electric field, and the arrows indicate the orientation of the field within the plane displayed. Note that the boundary between the two half-spaces is at a depth of $0 \mathrm{~m}$. Only the lower half-space, filled with water, is shown.

In the time slices, the diffusion of the electric field away from the source location can be seen as a high-amplitude (red) area that is expanding over time. We use the term diffusion front to name the zone where the amplitude of the electric field switches in one time step from undetectable to detectable in the next time step. This diffusion front, a function of space and time, is emphasized by the yellow curve, which highlights positions where the electric field has a strength of $10^{-14} \mathrm{~V} / \mathrm{m}$. Although in CSEM the electric field does not behave like a wave but like a diffusive field, it exhibits directionality, as indicated by the propagation of the diffusion front. This directionality is not related with the directionality from the vector nature of the electric field (white arrows). In contrast to a wavefield, the largest amplitude always remains at the source location. This is also the case for other source-time functions, such as the switch-on function (Heaviside step function) or the derivative of the Dirac delta function (Doublet function).

The electric field consists of a direct field and a reflected field, but no refracted energy - i.e., no airwave — is visible (Um and Alumbaugh, 2007) because a vertical source generates a TM-mode field only. The TE mode, which creates the airwave, is not present; therefore, the airwave is absent. In other words, an electric field created by a vertical source can be fully described by the last two terms of equation 1 . Precisely at the boundary, the vertical component of the electric field is zero, resulting in a perfectly horizontal electric-field vector at this location. The reason is the boundary condition, which demands that the vertical component of the electric current density $J=\sigma E$ be continuous across the interface (Nabighian, 1988). Because $J$ is zero in air, the vertical component of $J$ must be zero in the water at the boundary as well. Complete destructive interference between the vertical component of the direct and the reflected fields, from a TM-mode reflection coefficient of one, fulfills this boundary condition.

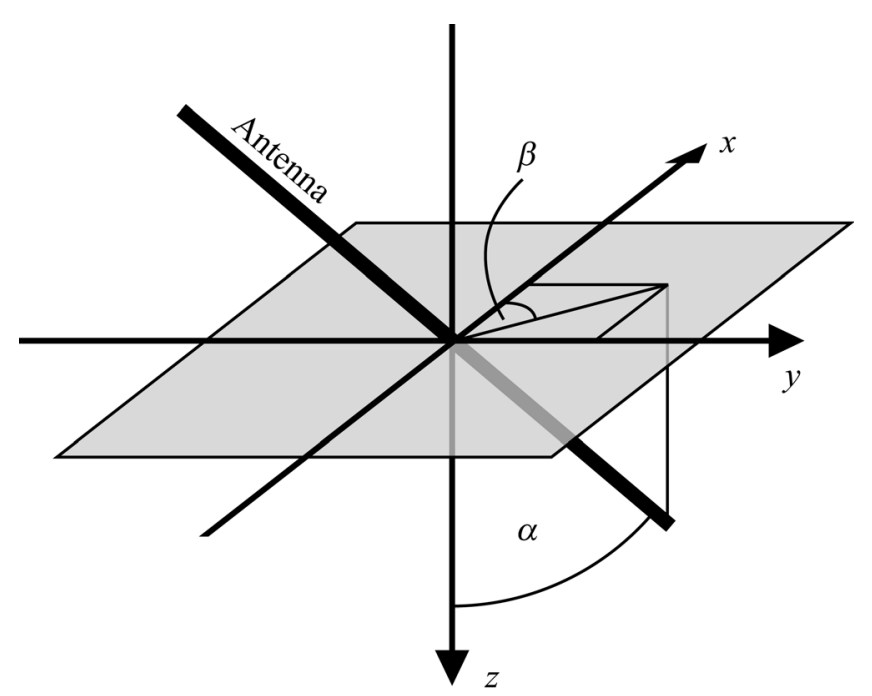

Figure 2. Relation between angles $\alpha$ (dip) and $\beta$ (azimuth). 
Figure 4 shows the diffusion of the electric field for the same setup but for a horizontally oriented source in the inline direction $\left(\alpha=90^{\circ}\right.$ and $\left.\beta=0^{\circ}\right)$. In this situation, the total field consists of the TE and TM modes; therefore, the airwave is expected to appear. The amplitude of the airwave starts to exceed $10^{-15} \mathrm{~V} / \mathrm{m}$ at the air-water interface at $10^{-2.45} \mathrm{~s}$ after the excitation of the source. It can be identified as an event diffusing downward almost simultaneously at all offsets. The horizontal decay, visible at shallow depth, is caused by the geometric spreading term proportional to the inverse horizontal distance cubed. In Figure $4 \mathrm{~b}$ and $4 \mathrm{c}$, showing the electric field at later times, the airwave forms the major contribution to the electric field at large offsets and shallow depth. The electricfield vector is perfectly horizontal in this area because the airwave is a pure TE mode and therefore has no vertical electric component. This goes hand in hand with the previous observation that a vertical source, which produces a pure TMmode field, does not feature the airwave. The airwave contribution has a late-time asymptote proportional to $t^{-3}$ but the direct field is proportional to $t^{-2.5}$.

\section{Elongated source}

In reality, the source antenna is not a point but has a physical extension. To check how our modeling results change with an elongated source, a 200-m-long source antenna was simulated by integration over the length of the antenna. Because of the
Figure 3. The diffusion of the electric field resulting from a vertical electric source in a halfspace of water $(\sigma=3 \mathrm{~S} / \mathrm{m})$ below a half-space of air $(\sigma=0 \mathrm{~S} / \mathrm{m})$ for different time steps: (a) $10^{-3} \mathrm{~s}$, (b) $10^{-2} \mathrm{~s}$, (c) $10^{-1} \mathrm{~s}$, (d) $10^{0} \mathrm{~s}$, and (e) $10^{1} \mathrm{~s}$ after the excitation of the source. The left column shows a vertical cross section in the inline direction at a crossline offset of $0 \mathrm{~m}$. The right column shows a horizontal cross section at a depth of $200 \mathrm{~m}$. The color stands for the amplitude of the total electric field between $10^{-15} \mathrm{~V} / \mathrm{m}$ (blue) and $10^{-8} \mathrm{~V} / \mathrm{m}$ (red). The arrows represent the orientation of the field within the plane shown. The yellow line indicates an amplitude of the electric field of $10^{-14} \mathrm{~V} / \mathrm{m}$.
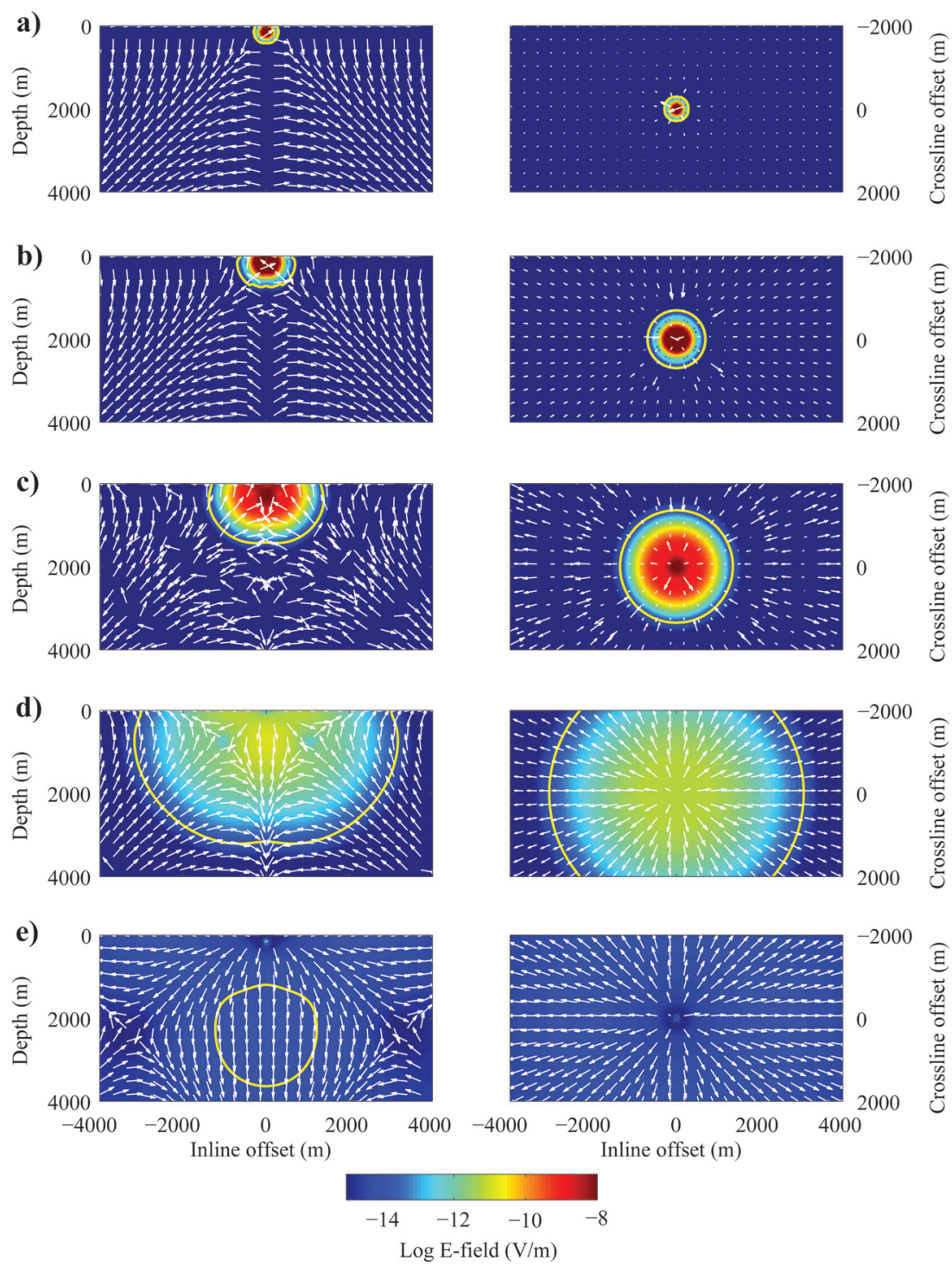
large computational cost of such an integration, this was only done for a few time steps. Figure 5 shows the electric field in the half-space of water for one of these time steps, which is $10^{-0.43} \mathrm{~s}$ after source excitation. The center of the source is located at a depth of $150 \mathrm{~m}$, and the antenna is dipping $20^{\circ}$ in the inline direction $\left(\alpha=20^{\circ}\right.$ and $\left.\beta=0^{\circ}\right)$.

In Figure $5 \mathrm{a}$, the electric field resulting from a point source is shown as in the previous figures; in Figure 5b, the source is 200 $\mathrm{m}$ long and the electric field has been normalized with the source-dipole moment to make the strength of the electric field independent of the antenna length. Because of the integration, some artifacts around the source location appear. Figure 5c shows the ratio of the electric field resulting from the point source to that from the elongated source of $200 \mathrm{~m}$. This ratio is approximately one almost everywhere except at kinks where the electric field changes direction abruptly. Consequently, the observations made for a point source are also valid for an elongated source because an elongated-source response is similar to a point-source response if the resulting field is normalized by the source-dipole moment.

\section{Effects of the airwave}

In this section, we investigate the effects of the airwave and its interference with the subsurface response, which includes the scattered field from the reservoir as well as the response from the ocean bottom for the case of an imperfect vertical source.
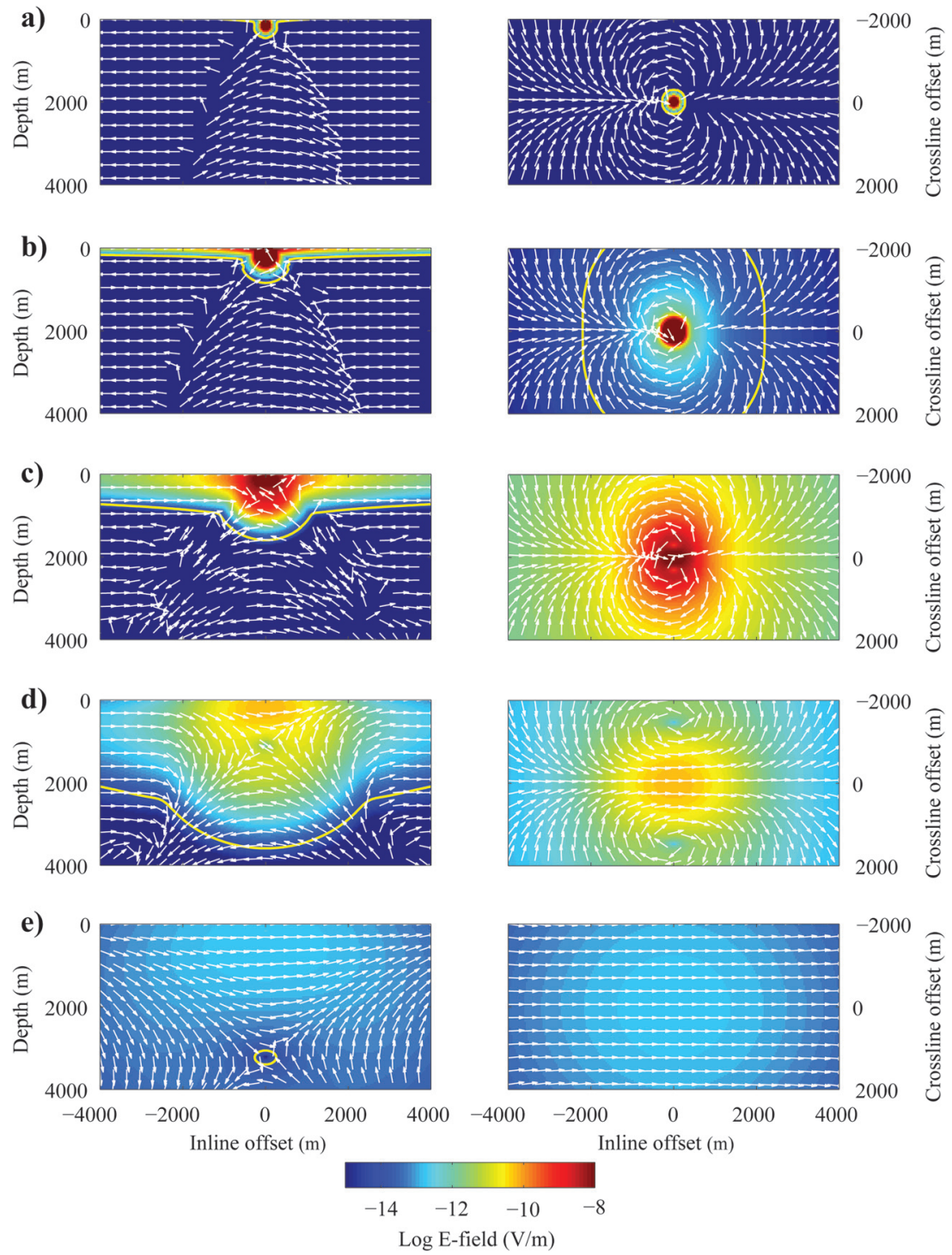

Figure 4. Same as Figure 3 but for a horizontal source. 
Therefore, we numerically model the electric field as a function of time and dip angle of the source for the situation plotted in Figure $1 \mathrm{~b}$ at three points. These three points are located at the ocean bottom at a depth of $200 \mathrm{~m}$ and at inline offsets of 500, 1000 , and $2000 \mathrm{~m}$. The source antenna dips between $0^{\circ}-\mathrm{a}$ perfect vertical source - and $1^{\circ}$. Assuming a length of $200 \mathrm{~m}$ for the vertical source antenna, a dip of $1^{\circ}$ means that the top point of the antenna is mispositioned by $3.5 \mathrm{~m}$.

Figure $6 a-6 c$ shows only the airwave at these three points. The airwave appears at all offsets at $10^{-2} \mathrm{~s}$ after the excitation of the source. Because the arrival time of the airwave depends only on the vertical distances between the source and the airwater interface and between the receiver and the air-water interface but not on the horizontal offset, the arrival time is the same for all three sample points. As a result of the geometric spreading of the electric field in the air, the amplitude of the airwave
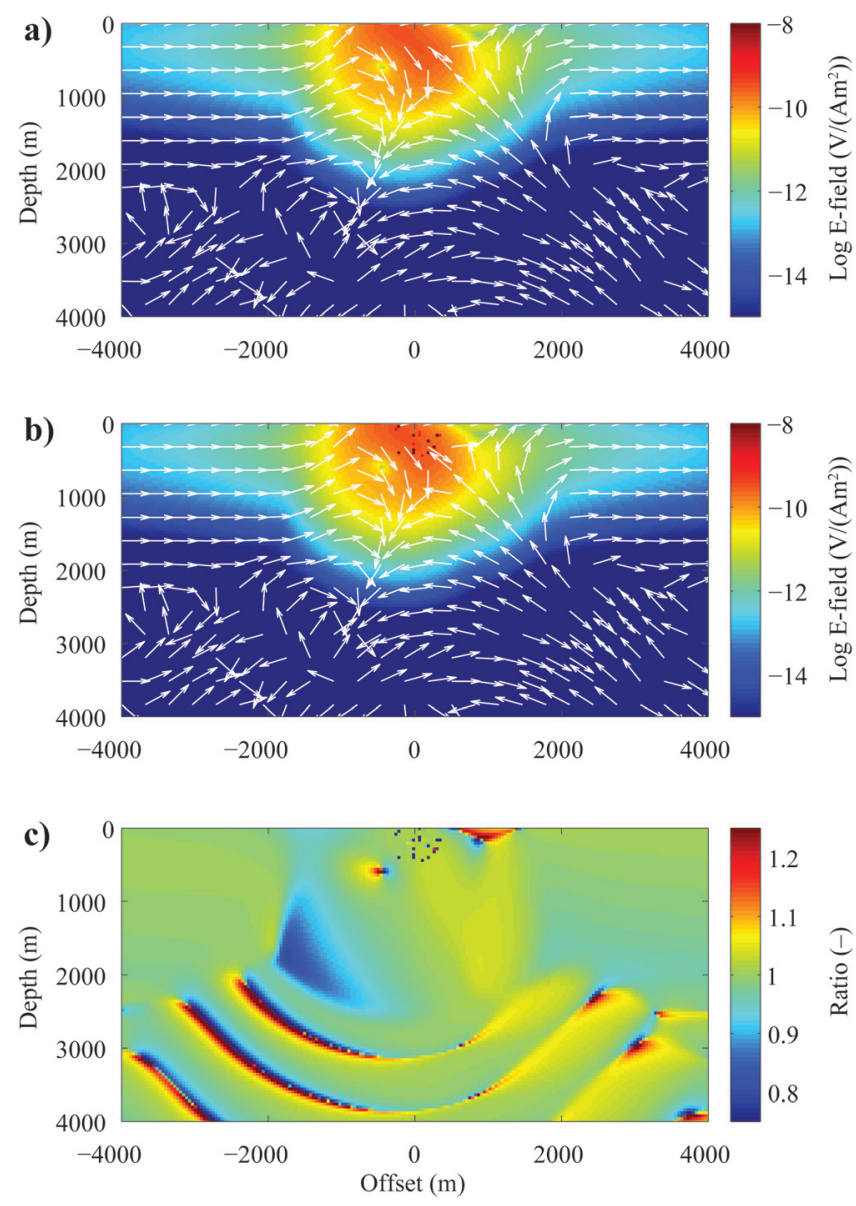

Figure 5. (a) Vertical cross section at a crossline offset of $0 \mathrm{~m}$, showing the electric field normalized by its source-dipole moment in the half-space of water $10^{-0.43} \mathrm{~s}$ after source excitation. The spatial-point source is located at a depth of $150 \mathrm{~m}$, dipping $20^{\circ}$ in the inline direction. The color represents the amplitude of the total electric field normalized by the dipole moment of the source on a common logarithmic scale $\left[\mathrm{V} /\left(\mathrm{Am}^{2}\right)\right]$. The arrows indicate the orientation of the field within the plane shown. (b) Same as (a), but the source antenna is $200 \mathrm{~m}$ long with its center at a depth of $150 \mathrm{~m}$. (c) The ratio of (a) point source to (b) long source on a linear scale. is offset-dependent. Only for perfect verticality of the source does the amplitude of the airwave remain below the detection level. As soon as the source is dipped slightly, the airwave appears with a significant amplitude.

Figure 6d-6f shows the airwave, the reflected field at the airwater interface, and the direct field in the water layer. Although the airwave is dependent on the dip angle of the source antenna, the direct and reflected fields seem to be invariant to the angles under consideration. With increasing offset, the contribution from the airwave separates in time from the rest of the field. The separation in time is possible because, on the one hand, the direct and reflected fields appear at later times with increasing offset, whereas the onset of the airwave remains invariant in time and, on the other hand, the airwave decays faster in time than the direct and reflected fields (Slob et al., 2010).

The complete field - the airwave, the reflected field, the direct field, and the subsurface response (i.e., the reservoir response and the response from the ocean bottom) - is shown in Figure 6g-6i. Similar to the direct field, the subsurface response is invariant to the angles under consideration. In the subsurface, especially in the reservoir, the diffusion front moves much faster than in the water layer because of the smaller conductivity. As a result of this and the shallow location of the reservoir, the subsurface response appears earlier than the direct field, leading to an overlap of the subsurface response with the airwave for our modeling setup (Figure 1b). In other words, the airwave hides the signal from the reservoir in our choice of reservoir depth and conductivity values. A better separation in time between the airwave and the reservoir response may be possible for a deeper reservoir.

The percentage of the airwave relative to the complete field, i.e., the field that contains all events, is shown in Figure $6 \mathrm{j}-61$. The contribution of the airwave at an offset of $2000 \mathrm{~m}$ for early times is almost $100 \%$ because it is basically the only component of the electric field at that moment. The direct and reflected fields appear later in time and are weaker in amplitude with increasing offset, so the airwave becomes more dominant at these large offsets. There is no airwave only for a perfect vertical source. As soon as there is a dip in the source, there is a contribution of the airwave to the complete electric field.

It is difficult to conclude how large of a percentage of airwave signal can be coped with, but it is possible to quantify the dip angle for a given airwave percentage. In other words, it is possible to plot the dip angle as a function of airwave percentage for specified offsets and time steps. These graphs are shown in Figure 7 for offsets of 500, 1000, and $2000 \mathrm{~m}$. These dip-angle-versus-airwave-percentage curves were computed for four different time steps at each offset location. All curves have the shape of an exponential function. This means that a large increase of airwave contribution happens for small dips of the source antenna. Because of the limited number of computed source dip angles, this steep increase appears as a step at $0 \%$ airwave contribution in the graph. If the source antenna already dips strongly, i.e., more than $50^{\circ}$ an additional dip causes little change in the airwave percentage. To ensure that the airwave is always smaller than $20 \%$ at all offsets, the source antenna should not be allowed to dip more than $0.05^{\circ}$. For a 200-m-long antenna, this corresponds to a positioning error of less than $0.2 \mathrm{~m}$. 

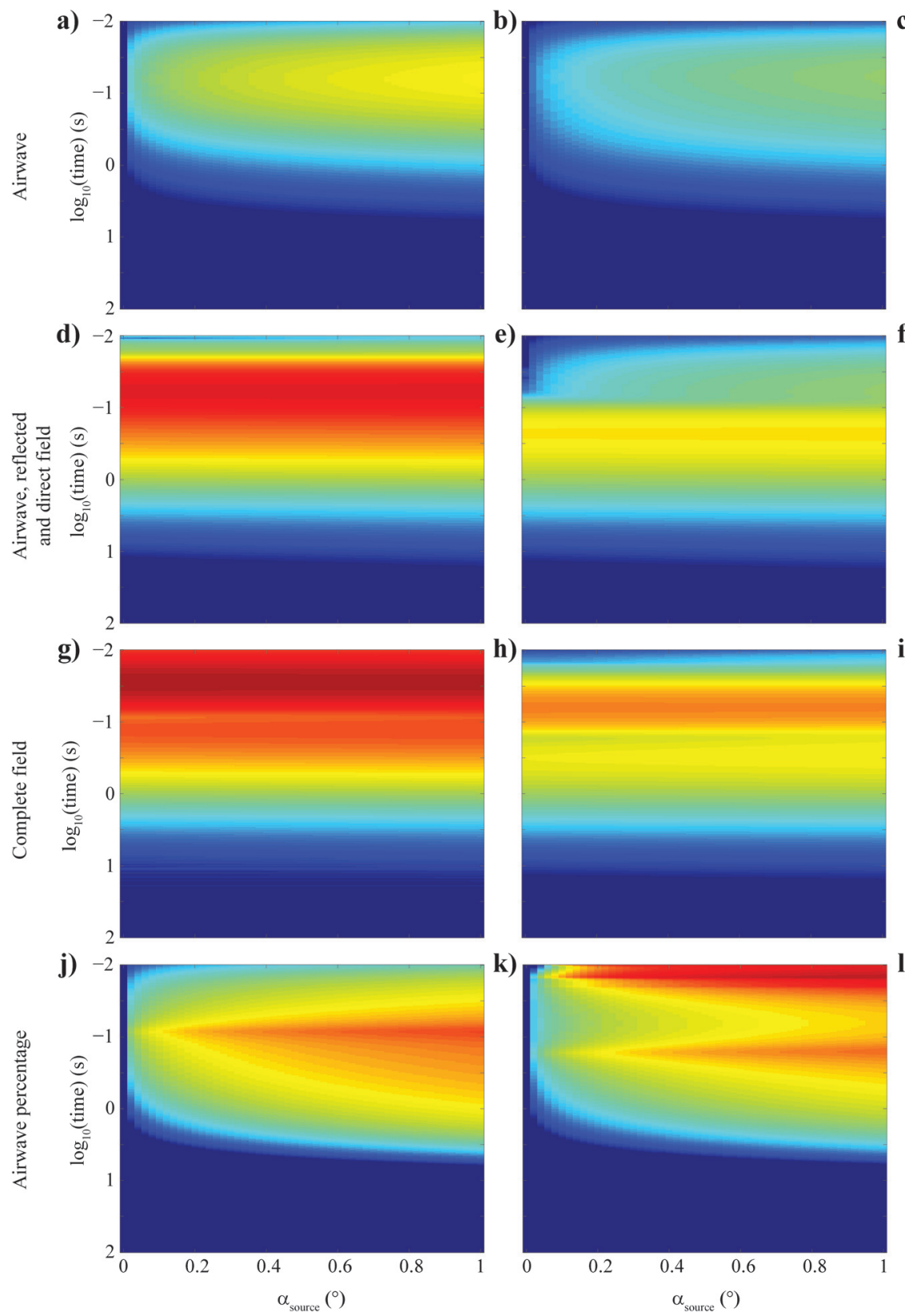
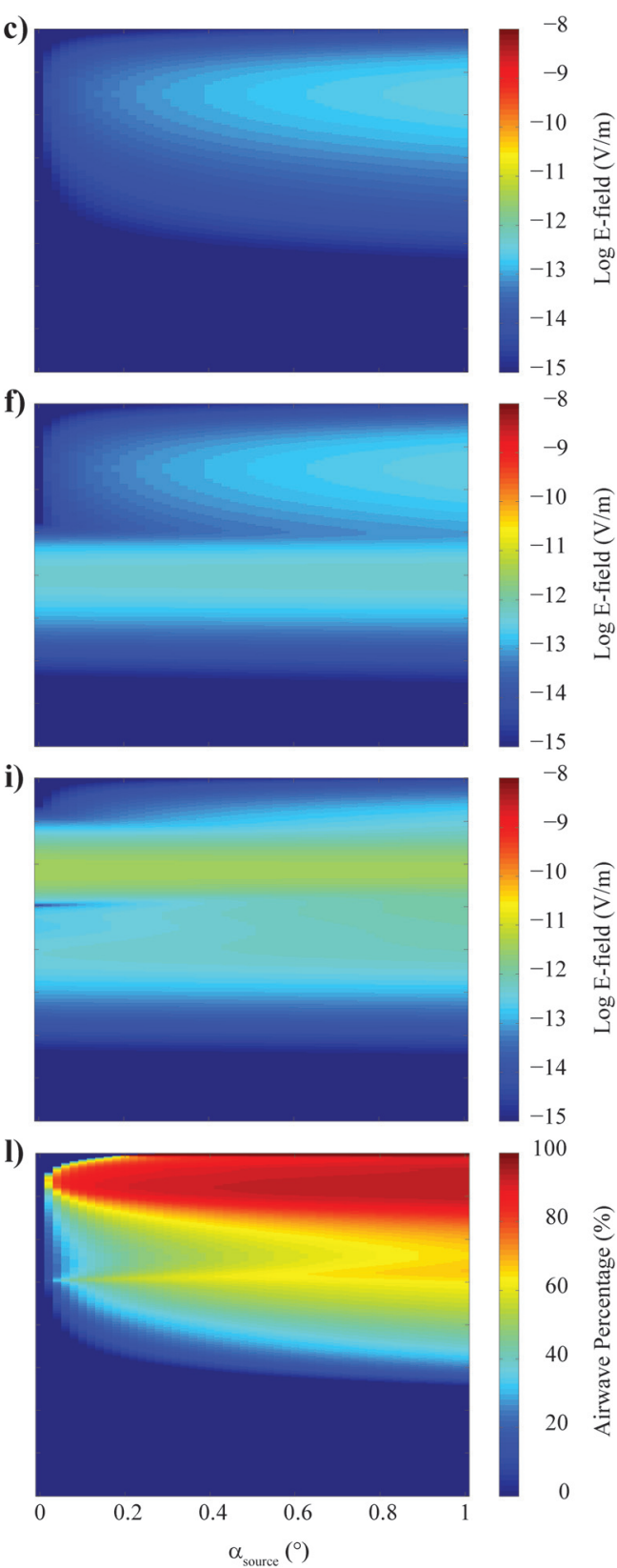

Figure 6. The electric field as a function of time and dip angle of the source for three points at a depth of $200 \mathrm{~m}$ and inline offsets of 500 , 1000, and $2000 \mathrm{~m}$. (Top row) Airwave only at offsets of (a) $500 \mathrm{~m}$, (b) $1000 \mathrm{~m}$, and (c) $2000 \mathrm{~m}$. (Second row) The airwave, reflected field, and direct field in the water at offsets of (d) $500 \mathrm{~m}$, (e) $1000 \mathrm{~m}$, and (f) $2000 \mathrm{~m}$. (g-i) Complete field, i.e., all events, including the response from the reservoir. (j-1) Percentage of airwave relative to the complete field. In $(\mathrm{a}-\mathrm{i})$, the color represents the amplitude of the total electric field on a common logarithmic scale. In (j-1), the color represents the percentage of the airwave on a linear scale.

a)

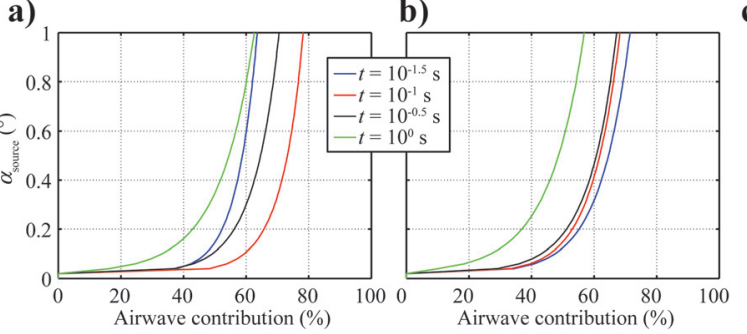

c)

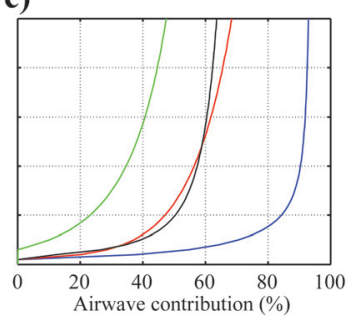

Figure 7. Source dip angle $\alpha_{\text {source }}$ as a function of the airwave percentage for four different time steps and three receiver locations: (a) $500 \mathrm{~m}$, (b) $1000 \mathrm{~m}$, and (c) $2000 \mathrm{~m}$. 


\section{CONCLUSIONS}

The directionality of wavefields is widely accepted, but the directionality of diffusive fields is often questioned. The development of the diffusive electromagnetic fields in space and time shows that diffusive fields exhibit directionality in the sense of a temporal and spatial expanding high-amplitude area, with the highest amplitude remaining at the source location. Consequently, if a diffusive field has a directionality comparable to a wavefield, techniques that thus far have been applied mostly to wavefields can be adapted for CSEM. Examples are decomposition into upward- and downward-decaying fields, decomposition and deconvolution of upward- by downward-decaying fields to obtain the CSEM impulse reflection response, and beam steering.

The amplitude of the electric field resulting from an elongated-source antenna is comparable with the amplitude of the electric field from a point-source antenna if normalized with the source-dipole moment, which is a constant factor. Consequently, observations made for a point source are also valid for an elongated source.

Vertical receivers are insensitive to the airwave, and a vertical source does not excite the airwave in a horizontally layered medium because the airwave has only horizontal components. Unfortunately, small deviations of the source antenna from verticality lead to a significant contribution of the airwave to the complete measured field, e.g., a dip of $0.05^{\circ}$ results in a contribution of the airwave of $20 \%$ with respect to the complete electric field, including the subsurface response. Still, for a horizontal source, the contribution of the airwave would be much larger. In our setup, the subsurface response appears at similar times as the airwave. The latter is thus masking a possible signal from a reservoir with a dipping source antenna. In that case, the airwave needs to be suppressed or separated in time-domain CSEM using similar methods as applied in frequency-domain CSEM. An airwave-removal scheme will usually result in fewer artifacts if the amplitude of the airwave is already relatively small. Consequently, it can still be advantageous to have an antenna dip slightly from verticality than a horizontally oriented antenna. For up/down separation or interferometry, multicomponent receivers are necessary.

\section{ACKNOWLEDGMENTS}

We thank Xinyou Lu, Rune Mittet, and two anonymous reviewers for their constructive comments, which significantly improved this manuscript. J. Hunziker would like to thank Roel Snieder for having him invited for a several-months' stay at Colorado School of Mines where part of this research has been done. This research is supported by the Dutch Technology Foundation (STW), the Applied Science division of the Netherlands Organization for Scientific Research (NWO), and the Technology Program of the Ministry of Economic Affairs.

\section{APPENDIX A}

\section{COEFFICIENTS, FUNCTIONS, AND PARAMETERS OF EQUATION 1}

The following equations are published for a VTI medium in Slob et al. (2010) and are shown here for the isotropic case. In this
Table A-1. Parameters used in Appendix A.

\begin{tabular}{lc} 
Description & Expression \\
\hline Conductivity & $\sigma$ \\
Free-space value of magnetic permeability & $\mu_{0}$ \\
Laplace transformation parameter & $s$ \\
Vertical wavenumber of & $\gamma=\sqrt{s \mu_{0} \sigma}$ \\
the lower half-space & $x_{3}, x_{3}^{S}$ \\
Receiver depth, source depth & $h^{ \pm}=\left|x_{3} \pm x_{3}^{S}\right|$ \\
Vertical distance from source to receiver & $\rho=\sqrt{x_{1}^{2}+x_{2}^{2}}$ \\
Horizontal distance in space & $r^{ \pm}=\sqrt{\rho^{2}+\left(h^{ \pm}\right)^{2}}$ \\
Radial distance from source to receiver & $t$ \\
Time & $\tau^{ \pm}=\frac{\sigma \mu_{0}\left(r^{ \pm}\right)^{2}}{4}$ \\
Offset-dependent diffusion time &
\end{tabular}

section, we use subscript notation. The coordinate vector $\mathbf{x}$ is therefore $\left(x_{1}, x_{2}, x_{3}\right)^{T}$ where the superscript $\mathrm{T}$ stands for transposition. Table A-1 lists all parameters required in the following coefficients and functions.

The TM-mode coefficients for the horizontal components are given by

$$
\begin{gathered}
g_{\alpha \beta ; 0}^{\mathrm{TM} ; \pm}=\frac{3 x_{\alpha} x_{\beta}-\left(r^{ \pm}\right)^{2} \delta_{\alpha \beta}}{4 \pi \sigma\left(r^{ \pm}\right)^{5}}, \\
g_{\alpha \beta ; \pm}^{\mathrm{TM} ; \pm}=\left(\frac{3 x_{\alpha} x_{\beta}-\left(r^{ \pm}\right)^{2} \delta_{\alpha \beta}}{4 \pi\left(r^{ \pm}\right)^{4}}-\frac{2 x_{\alpha} x_{\beta}-\rho^{2} \delta_{\alpha \beta}}{4 \pi \rho^{4}}\right) \frac{\sqrt{\mu_{0}}}{\sqrt{\sigma}},
\end{gathered}
$$

and

$$
g_{\alpha \beta ; 2}^{\mathrm{TM} ; \pm}=\frac{\mu_{0} x_{\alpha} x_{\beta}}{4 \pi r^{ \pm}}\left(\frac{1}{\left(r^{ \pm}\right)^{2}}-\frac{1}{\rho^{2}}\right)
$$

where the subscripts $\alpha$ and $\beta$ can have the values one or two, representing the horizontal components. The Kronecker delta $\delta_{\alpha \beta}$ is one for $\alpha=\beta$ and zero otherwise. The TE-mode coefficients for the horizontal components are given by

$$
\begin{gathered}
g_{\alpha \beta ; 0}^{\mathrm{TE} ;-}=0, \\
g_{\alpha \beta ; 0}^{\mathrm{TE} ;+}=\frac{3 f_{\alpha \beta}-\left(r^{+}\right)^{2} \delta_{\alpha \beta}}{2 \pi \sigma\left(r^{+}\right)^{5}}, \\
g_{\alpha \beta ; 1}^{\mathrm{TE} ;-}=\frac{x_{\alpha} x_{\beta}-f_{\alpha \beta}}{4 \pi \rho^{4}} \frac{\sqrt{\mu_{0}}}{\sqrt{\sigma}},
\end{gathered}
$$

$$
\begin{gathered}
g_{\alpha \beta ; 1}^{\mathrm{TE} ;+}=\left(\frac{3 f_{\alpha \beta}-\left(r^{+}\right)^{2} \delta_{\alpha \beta}}{2 \pi \sqrt{\sigma}\left(r^{+}\right)^{4}}+\frac{x_{\alpha} x_{\beta}-f_{\alpha \beta}}{4 \pi \rho^{4}}\right) \frac{\sqrt{\mu_{0}}}{\sqrt{\sigma}}, \\
g_{\alpha \beta ; 2}^{\mathrm{TE} ;-}=-\frac{\mu_{0} f_{\alpha \beta}}{4 \pi \rho^{2} r^{-}},
\end{gathered}
$$


and

$$
g_{\alpha \beta ; 2}^{\mathrm{TE} ;+}=\frac{\mu_{0} f_{\alpha \beta}}{4 \pi r^{+}}\left(\frac{2}{\left(r^{+}\right)^{2}}-\frac{1}{\rho^{2}}\right),
$$

where $f_{\alpha \beta}=\left(\rho^{2} \delta_{\alpha \beta}-x_{\alpha} x_{\beta}\right)$. For the vertical components, only TM-mode coefficients are nonzero:

$$
\begin{gathered}
g_{3 \alpha ; 0}^{\mathrm{TM} ;-}=\frac{3 x_{\alpha}\left(x_{3}-x_{3}^{S}\right)}{4 \pi \sigma\left(r^{-}\right)^{5}}, \\
g_{3 \alpha ; 0}^{\mathrm{TM} ;+}=\frac{3 x_{\alpha} h^{+}}{4 \pi \sigma\left(r^{+}\right)^{5}}, \\
g_{3 \alpha ; 1}^{\mathrm{TM} ;-}=\frac{3 x_{\alpha}\left(x_{3}-x_{3}^{S}\right)}{4 \pi\left(r^{-}\right)^{4}} \frac{\sqrt{\mu_{0}}}{\sqrt{\sigma}}, \\
g_{3 \alpha ; 1}^{\mathrm{TM} ;+}=\frac{3 x_{\alpha} h^{+}}{4 \pi\left(r^{+}\right)^{4}} \frac{\sqrt{\mu_{0}}}{\sqrt{\sigma}}, \\
g_{3 \alpha ; 2}^{\mathrm{TM} ;-}=\mu_{0} \frac{x_{\alpha}\left(x_{3}-x_{3}^{S}\right)}{4 \pi\left(r^{-}\right)^{3}},
\end{gathered}
$$

and

$$
g_{3 \alpha ; 2}^{\mathrm{TM} ;+}=\mu_{0} \frac{x_{\alpha} h^{+}}{4 \pi\left(r^{+}\right)^{3}} .
$$

In view of reciprocity,

$$
\begin{gathered}
g_{\alpha 3 ; m}^{\mathrm{TM} ;-}=g_{3 \alpha ; m}^{\mathrm{TM} ;-}, \\
g_{\alpha 3 ; m}^{\mathrm{TM} ;+}=-g_{3 \alpha ; m}^{\mathrm{TM} ;+} .
\end{gathered}
$$

The last coefficients are found as

$$
\begin{array}{r}
g_{33 ; 0}^{\mathrm{TM} ; \pm}=\mp \frac{\left(3\left(h^{ \pm}\right)^{2}-\left(r^{ \pm}\right)^{2}\right)}{4 \pi \sigma\left(r^{ \pm}\right)^{5}}, \\
g_{33 ; 1}^{\mathrm{TM} ; \pm}=\mp \frac{\left(3\left(h^{ \pm}\right)^{2}-\left(r^{ \pm}\right)^{2}\right)}{4 \pi\left(r^{ \pm}\right)^{4}} \frac{\sqrt{\mu_{0}}}{\sqrt{\sigma}},
\end{array}
$$

and

$$
g_{33 ; 2}^{\mathrm{TM} ; \pm}=\mp \mu_{0} \frac{\left(\left(h^{ \pm}\right)^{2}-\left(r^{ \pm}\right)^{2}\right)}{4 \pi\left(r^{ \pm}\right)^{3}} .
$$

The diffusion functions are given by

$$
\begin{aligned}
& F_{m}^{-}=F_{m}\left(t, \tau^{-}\right), \\
& F_{m}^{+}=F_{m}\left(t, \tau^{+}\right),
\end{aligned}
$$

where

$$
F_{0}(t, \tau)=\sqrt{\frac{\tau}{\pi t^{3}}} \exp \left(\frac{-\tau}{t}\right) H(t)
$$

$$
F_{1}(t, \tau)=\left(\frac{\tau}{t}-\frac{1}{2}\right) \sqrt{\frac{1}{\pi t^{3}}} \exp \left(\frac{-\tau}{t}\right) H(t),
$$

and

$$
F_{2}(t, \tau)=\left(\frac{\tau}{t}-\frac{3}{2}\right) \sqrt{\frac{\tau}{\pi t^{5}}} \exp \left(\frac{-\tau}{t}\right) H(t) .
$$

Here, $H(t)$ denotes the unit step function. The horizontal components of the term $P_{k r}$ are given by

$$
\begin{aligned}
P_{\alpha \beta}= & \frac{\sigma \mu_{0}^{2} h^{+} \exp \left(\frac{-\tau_{h}^{+}}{t}\right)}{32 \pi t^{3}}\left\{2\left(\delta_{\alpha \beta}-\frac{x_{\alpha} x_{\beta}}{\rho^{2}}\right) \bar{I}_{1}\left(\frac{\tau_{\rho}}{t}\right)\right. \\
& \left.-\left(\frac{\sigma \mu_{0}}{2 t}\left(\rho^{2} \delta_{\alpha \beta}-x_{\alpha} x_{\beta}\right)-\delta_{\alpha \beta}\right)\left(\bar{I}_{0}\left(\frac{\tau_{\rho}}{t}\right)-\bar{I}_{1}\left(\frac{\tau_{\rho}}{t}\right)\right)\right\},
\end{aligned}
$$

where $\tau_{h}^{+}=\sigma \mu_{0}\left(h^{+}\right)^{2} / 4$ and $\tau_{\rho}=\sigma \mu_{0} \rho^{2} / 8$. The scaled modified Bessel functions $\bar{I}_{0}$ and $\bar{I}_{1}$ are related to the modified Bessel functions $I_{0}$ and $I_{1}$ as follows:

$$
\bar{I}_{n}\left(\frac{\tau_{\rho}}{t}\right)=\exp \left(-\frac{\tau_{\rho}}{t}\right) I_{n}\left(\frac{\tau_{\rho}}{t}\right) .
$$

All other airwave terms, i.e., $P_{3 \alpha} P_{\alpha 3}$ and $P_{33}$ are zero.

In equation 1 , we use coefficients for the TE and TM modes. In an isotropic medium, the diffusion functions are the same for both modes, so equation 1 can be simplified to

$$
E_{k r}=P_{k r}+\sum_{m=0}^{2}\left(g_{k r ; m}^{-} F_{m}^{-}+g_{k r ; m}^{+} F_{m}^{+}\right),
$$

with the coefficients

$$
g_{k r ; 0}^{-}=\frac{3\left(x_{k}-x_{k}^{S}\right)\left(x_{r}-x_{r}^{S}\right)-\left(r^{-}\right)^{2} \delta_{k r}}{4 \pi \sigma\left(r^{-}\right)^{5}},
$$

$$
g_{k r ; 1}^{-}=\frac{3\left(x_{k}-x_{k}^{S}\right)\left(x_{r}-x_{r}^{S}\right)-\left(r^{-}\right)^{2} \delta_{k r}}{4 \pi\left(r^{-}\right)^{4}} \frac{\sqrt{\mu_{0}}}{\sqrt{\sigma}},
$$

$$
g_{k r ; 2}^{-}=\frac{\left(x_{k}-x_{k}^{S}\right)\left(x_{r}-x_{r}^{S}\right)-\left(r^{-}\right)^{2} \delta_{k r}}{4 \pi\left(r^{-}\right)^{3}} \mu_{0},
$$

$$
g_{\alpha \beta ; 0}^{+}=-3 \frac{x_{\alpha} x_{\beta}+\left(\left(r^{+}\right)^{2}-2 \rho^{2}\right) \delta_{\alpha \beta}}{4 \pi \sigma\left(r^{+}\right)^{5}},
$$

$$
g_{\alpha \beta ; 1}^{+}=-3 \frac{x_{\alpha} x_{\beta}+\left(\left(r^{+}\right)^{2}-2 \rho^{2}\right) \delta_{\alpha \beta}}{4 \pi\left(r^{+}\right)^{4}} \frac{\sqrt{\mu_{0}}}{\sqrt{\sigma}},
$$

$$
g_{\alpha \beta ; 2}^{+}=-\frac{x_{\alpha} x_{\beta}+\left(\left(r^{+}\right)^{2}-2 \rho^{2}\right) \delta_{\alpha \beta}}{4 \pi\left(r^{+}\right)^{3}} \mu_{0},
$$

$$
g_{k 3 ; 0}^{+}=-\frac{3\left(x_{k}+x_{k}^{S}\right)\left(x_{3}+x_{3}^{S}\right)-\left(r^{+}\right)^{2} \delta_{k 3}}{4 \pi \sigma\left(r^{+}\right)^{5}},
$$

$$
g_{k 3 ; 1}^{+}=-\frac{3\left(x_{k}+x_{k}^{S}\right)\left(x_{3}+x_{3}^{S}\right)-\left(r^{+}\right)^{2} \delta_{k 3}}{4 \pi\left(r^{+}\right)^{4}} \frac{\sqrt{\mu_{0}}}{\sqrt{\sigma}},
$$


Figure B-1. Relative error for the airwave term $P_{11}$ using (a) early (equations B-1 and B-2 ), (b) late (equations B-3 and B-4), and (c) mixed-time approximations for $h^{+}=500 \mathrm{~m}$

$$
\begin{gathered}
g_{k 3 ; 2}^{+}=-\frac{\left(x_{k}+x_{k}^{S}\right)\left(x_{3}+x_{3}^{S}\right)-\left(r^{+}\right)^{2} \delta_{k 3}}{4 \pi\left(r^{+}\right)^{3}} \mu_{0}, \\
g_{3 \beta ; 0}^{+}=\frac{3 x_{\beta}\left(x_{3}+x_{3}^{S}\right)}{4 \pi \sigma\left(r^{+}\right)^{5}}, \\
g_{3 \beta ; 1}^{+}=\frac{3 x_{\beta}\left(x_{3}+x_{3}^{S}\right)}{4 \pi\left(r^{+}\right)^{4}} \frac{\sqrt{\mu_{0}}}{\sqrt{\sigma}},
\end{gathered}
$$

and

$$
g_{3 \beta ; 2}^{+}=\frac{x_{\beta}\left(x_{3}+x_{3}^{S}\right)}{4 \pi\left(r^{+}\right)^{3}} \mu_{0} .
$$

From this, we arrive at the delta function response:

$$
\begin{aligned}
E_{k r}= & P_{k r} \\
& +\left(\frac{\sigma \mu_{0}}{4 \pi t}\right)^{3 / 2}\left(\frac{\delta_{k r}}{\sigma t}+\frac{\left(x_{k}-x_{k}^{S}\right)\left(x_{r}-x_{r}^{S}\right)-\left(r^{-}\right)^{2} \delta_{k r}}{4 t^{2}} \mu_{0}\right) \\
& \times \exp \left(-\frac{\tau^{-}}{t}\right)-\left(\frac{\sigma \mu_{0}}{4 \pi t}\right)^{3 / 2} \delta_{k \alpha} \delta_{r \beta} \\
& \times\left(\frac{x_{\alpha} x_{\beta}+\left(\left(r^{+}\right)^{2}-2 \rho^{2}\right) \delta_{\alpha \beta}}{4 t^{2}}\right) \mu_{0} \exp \left(-\frac{\tau^{+}}{t}\right) \\
& -\left(\frac{\sigma \mu_{0}}{4 \pi t}\right)^{3 / 2} \delta_{r 3}\left(\frac{\delta_{k 3}}{\sigma t}+\frac{\left(x_{k}+x_{k}^{S}\right)\left(x_{3}+x_{3}^{S}\right)-\left(r^{+}\right)^{2} \delta_{k 3}}{4 t^{2}} \mu_{0}\right) \\
& \times \exp \left(-\frac{\tau^{+}}{t}\right)+\left(\frac{\sigma \mu_{0}}{4 \pi t}\right)^{3 / 2} \delta_{r \beta} \delta_{k 3} \frac{x_{\beta}\left(x_{3}+x_{3}^{S}\right)}{4 t^{2}} \mu_{0} \\
& \times \exp \left(-\frac{\tau^{+}}{t}\right) .
\end{aligned}
$$

\section{APPENDIX B}

\section{APPROXIMATIONS OF THE AIRWAVE TERM}

Although we use the complete expression for the airwave in our computation, approximations are possible. For early times, i.e., $t \ll \tau_{\rho}$ or in other words for large arguments, the Bessel functions $\bar{I}_{n}$ in equation A-26 can be approximated by (Abramowitz and Stegun, 1972)

$$
\bar{I}_{0}\left(\frac{\tau_{\rho}}{t}\right) \approx \frac{1}{\sqrt{2 \pi \frac{\tau_{\rho}}{t}}}\left\{1+\frac{t}{8 \tau_{\rho}}+\frac{9}{2}\left(\frac{t}{8 \tau_{\rho}}\right)^{2}+\frac{75}{2}\left(\frac{t}{8 \tau_{\rho}}\right)^{3}\right\}
$$

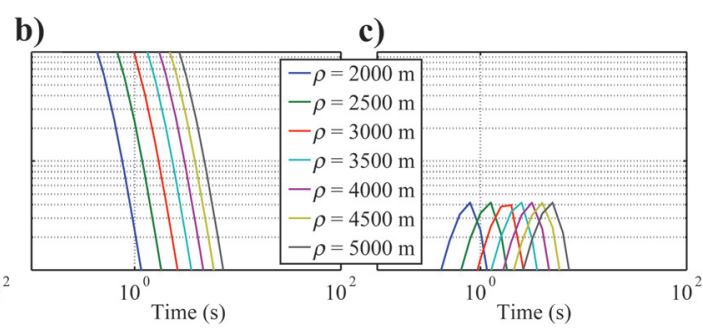

$$
\bar{I}_{1}\left(\frac{\tau_{\rho}}{t}\right) \approx \frac{1}{\sqrt{2 \pi \frac{\tau_{\rho}}{t}}}\left\{1-3 \frac{t}{8 \tau_{\rho}}-\frac{15}{2}\left(\frac{t}{8 \tau_{\rho}}\right)^{2}-\frac{105}{2}\left(\frac{t}{8 \tau_{\rho}}\right)^{3}\right\}
$$

Figure B-1a shows the normalized error for the airwave term $P_{11}$ using equations B-1 and B-2 to replace the Bessel functions $\bar{I}_{n}$ in equation A-26. The relative error is plotted for seven different horizontal distances $\rho$.

For late times, i.e., $t \gg \tau_{\rho}$ (for small arguments), the Bessel functions $\bar{I}_{n}$ can be approximated as (Abramowitz and Stegun, 1972)

$$
\begin{aligned}
& \bar{I}_{0}\left(\frac{\tau_{\rho}}{t}\right) \approx \exp \left(-\frac{\tau_{\rho}}{t}\right)\left\{1+\left(\frac{\tau_{\rho}}{2 t}\right)^{2}+\frac{1}{4}\left(\frac{\tau_{\rho}}{2 t}\right)^{4}\right\} \\
& \bar{I}_{1}\left(\frac{\tau_{\rho}}{t}\right) \approx \exp \left(-\frac{\tau_{\rho}}{t}\right) \frac{\tau_{\rho}}{2 t}\left\{1+\frac{1}{2}\left(\frac{\tau_{\rho}}{2 t}\right)^{2}+\frac{1}{12}\left(\frac{\tau_{\rho}}{2 t}\right)^{4}\right\} .
\end{aligned}
$$

The relative error for the airwave term $P_{11}$ using equations B-3 and B-4 to replace the Bessel functions $\bar{I}_{n}$ in equation A-26 is shown in Figure B-1b. The two approximations are combined such that equations B-1 and B-2 are used if $t<\left(\tau_{\rho} / 2\right)$ and equations B-3 and B-4 if $t>\left(\tau_{\rho} / 2\right)$. The relative error for this combination is plotted in Figure B-1c. At early and late times, the error is small, but it peaks as expected at intermediate times. The largest error is approximately $4 \%$.

\section{REFERENCES}

Abramowitz, M., and I. A. Stegun, 1972, Handbook of mathematical functions, 10th ed.: National Bureau of Standards.

Amundsen, L., L. Løseth, R. Mittet, S. Ellingsrud, and B. Ursin, 2006, Decomposition of electromagnetic fields into upgoing and downgoing components: Geophysics, 71, no. 5, G211-G223, doi:10.1190/ 1.2245468 .

Bannister, P. R., 1984, New simplified formulas for ELF subsurface-tosubsurface propagation: IEEE Journal of Oceanic Engineering, 9, no. 3, 154-163, doi:10.1109/JOE.1984.1145620.

Chave, A. D., S. C. Constable, and R. N. Edwards, 1991, Electrical exploration methods for the seafloor, in $\mathrm{M}$. Nabighian, ed., Electromagnetic methods in applied geophysics: SEG, 931-966.

Chen, J., and D. L. Alumbaugh, 2011, Three methods for mitigating airwaves in shallow water marine controlled-source electromagnetic data: Geophysics, 76, no. 2, F89-F99, doi:10.1190/1.3536641.

Constable, S., 2010, Ten years of marine CSEM for hydrocarbon exploration: Geophysics, 75, no. 5, 75A67-75A81, doi:10.1190/1.3483451.

Constable, S., and C. J. Weiss, 2006, Mapping thin resistors and hydrocarbons with marine EM methods: Insights from 1D modeling: Geophysics, 71, no. 2, G43-G51, doi:10.1190/1.2187748.

Holten, T., E. G. Flekkøy, B. Singer, E. M. Blixt, A. Hanssen, and K. J. Måløy, 2009, Vertical source, vertical receiver, electromagnetic technique for offshore hydrocarbon exploration: First Break, 27, 89-93.

King, R. W., M. Owens, and T. Wu, 1992, Lateral electromagnetic waves, theory and applications to communications, geophysical exploration, and remote sensing: Springer-Verlag New York, Inc. 
Løseth, L. O., L. Amundsen, and A. J. K. Jenssen, 2010, A solution to the airwave-removal problem in shallow-water marine EM: Geophysics, 75, no. 5, A37-A42, doi:10.1190/1.3475359.

Maaø, F. A., and A. K. Nguyen, 2010, Enhanced subsurface response for marine CSEM surveying: Geophysics, 75, no. 3, A7-A10, doi:10.1190/ 1.3377054 .

Mittet, R., 2008, Normalized amplitude ratios for frequency-domain CSEM in very shallow water: First Break, 26, 47-54.

Mulder, W. A., 2006, A multigrid solver for 3D electromagnetic diffusion: Geophysical Prospecting, 54, 633-649, doi:10.1111/j.1365-2478 2006.00558.x.

Mulder, W. A., M. Wirianto, and E. C. Slob, 2008, Time-domain modeling of electromagnetic diffusion with a frequency-domain code: Geophysics, 73, no. 1, F1-F8, doi:10.1190/1.2799093.

Nabighian, M. N., 1988, Electromagnetic methods in applied geophysics: SEG.

Nordskag, J. I., and L. Amundsen, 2007, Asymptotic airwave modeling for marine controlled-source electromagnetic surveying: Geophysics, 72, no. 6, F249-F255, doi:10.1190/1.2786025.

Raiche, A. P., and J. H. Coggon, 1975, Analytic Green's tensors for integral equation modelling: Geophysical Journal of the Royal Astronomical Society, 42, 1035-1038, doi:10.1111/j.1365-246X.1975. tb06465.x.
Slob, E., J. Hunziker, and W. Mulder, 2010, Green's tensors for the diffusive electric field in a VTI half-space: Progress in Electromagnetics Research, 107, 1-20, doi:10.2528/PIER10052807.

Um, E. S., and D. L. Alumbaugh, 2007, On the physics of the marine controlled-source electromagnetic method: Geophysics, 72, no. 2, WA13WA26, doi:10.1190/1.2432482.

Wait, J. R., 1960, Propagation of electromagnetic pulses in a homogeneous conducting earth: Applied Scientific Research, 8, 213-253, doi: 10.1007/BF0292478.

Wapenaar, K., E. Slob, and R. Snieder, 2008, Seismic and electromagnetic controlled-source interferometry in dissipative media: Geophysical Prospecting, 56, no. 3, 419-434, doi:10.1111/j.1365-2478.2007.00686.x.

Weiss, C. J., 2007, The fallacy of the "shallow-water problem" in marine CSEM exploration: Geophysics, 72, no. 6, A93-A97, doi:10.1190/ 1.2786868 .

Wirianto, M., W. A. Mulder, and E. C. Slob, 2011, Exploiting the airwave for time-lapse reservoir monitoring with CSEM on land: Geophysics, 76, no. 3, A15-A19, doi:10.1190/1.3560157.

Ziolkowski, A., 2007, Developments in the transient electromagnetic method: First Break, 25, 99-106.

Ziolkowski, A., and D. Wright, 2007, Removal of the airwave in shallow marine transient EM data: 77th Annual International Meeting, SEG, Expanded Abstracts, 534-538, doi: 10.1190/1.2792478. 